\title{
Concept of modes in optics and photonics
}

\section{Rene Daendliker}

Rene Daendliker, "Concept of modes in optics and photonics," Proc. SPIE 3831, Sixth International Conference on Education and Training in Optics and Photonics, (16 June 2000); doi: 10.1117/12.388718 


\title{
The concept of modes in optics and photonics
}

\author{
René Dändliker \\ Institute of Microtechnology, University of Neuchâtel, CH-2000 Neuchâtel, Switzerland*
}

\begin{abstract}
The concept of modes, or eigenfunctions, is fundamental for all wave phenomena in physics like optics, acoustics and quantum mechanics. In optics and photonics, the concept of modes is well suited to describe emission and absorption, coherence and interference, propagation and dispersion. The concept of modes consists of two aspects: first, the modes are solutions for the propagation of the light; second, the number of photons in the different modes describes the transport of energy or information. The energy density of the black body radiation, given by Planck's law, is the product of the density of modes in free space and the average number of photons per mode, given by the Bose-Einstein distribution. The transverse shape of the modes is determined by diffraction and boundary conditions. The longitudinal extension is given by the coherence length.
\end{abstract}

Keywords: modes, eigenfunctions, diffraction, coherence, interference, dispersion, waveguides, Planck's law, lasers

\section{INTRODUCTION}

The concept of modes, or eigenfunctions, is fundamental for all wave phenomena in physics like optics, acoustics and quantum mechanics. In optics and photonics, the concept of modes is well suited to describe emission and absorption, coherence and interference, propagation and dispersion.

The concept of modes consists of two aspects: first, the modes are solutions for the propagation of the light; second, the number of photons in the different modes describes the transport of energy or information. The energy density of the black body radiation, given by Planck's law, is the product of the density of modes in free space and the average number of photons per mode, given by the Bose-Einstein distribution. The transverse shape of the modes is determined by diffraction and boundary conditions. The longitudinal extension is given by the coherence length. They following properties and laws are related to modes:

- Modes are orthogonal; they do not interfere.

- Only the light (photons) within one and the same mode is coherent and does interfere (for identical polarization).

- Different modes may have different phase and group velocities (dispersion).

- The quality of light is given by the number $\mathrm{N}$ of photons per mode: $\mathrm{N} \ll 1$ for thermal sources and $\mathrm{N} \gg 1$ for lasers.

- The shape of modes may be changed by passive optical elements (lenses, mirrors, filters), however, the number $\mathrm{N}$ of photons per mode cannot by increased.

- The energy throughput of an optical systems depends on the number of supported modes and the adaptation of the modes between the different sections of the system.

- Noise in coherent electro-optical detection depends on the number of photons per mode.

These are the reasons why I believe that introducing the concept of modes is most adequate for education in optics and photonics at least at a university level.

\section{DEFINITION OF MODES}

The modes are basically defined by the properties of coherence and orthogonality: modes are orthogonal solutions of the wave equation, they do not interfere (the energy or optical power of a linear superposition of modes is equal to the sum the energy or the optical power of the individual modes). Only the light (photons) within one and the same mode is coherent and does interfere. Two types of modes are distinguished: spatial modes transverse to the direction of propagation (crosssection and divergence) and temporal modes in the direction of propagation (time and frequency).

\footnotetext{
* Correspondence: Email: rene.dandliker@imt.unine.ch; WWW: http://www-optics.unine.ch
} 


\subsection{Spatial modes}

Spatial modes are defined by the diffraction limit. The prototype of a spatial mode is the Gaussian beam, shown in Fig. 1.

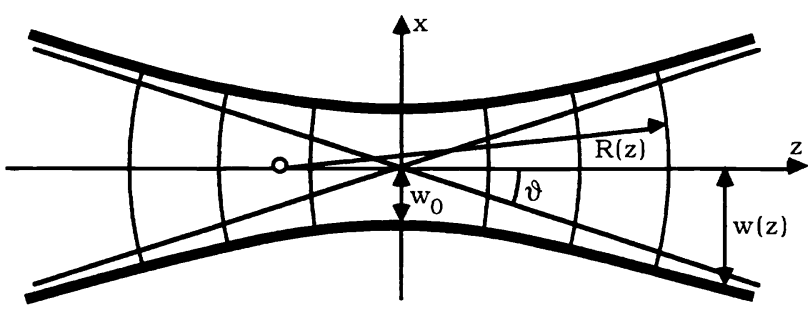

Fig. 1. Gaussian beam as prototype of a spatial mode.
The diffraction limited divergence $\vartheta$ is given by

$$
\vartheta=\lim _{z \rightarrow \infty} \frac{w(z)}{z}=\frac{\lambda}{\pi w_{0}},
$$

where $\lambda$ is the optical wavelength. In two dimensions we get

$$
\mathrm{A}_{\mathrm{M}}=\pi \mathrm{w}_{0}{ }^{2} \quad \text { and } \quad \Omega_{\mathrm{M}}=\pi \vartheta^{2}
$$

for the cross-sectional area $A_{M}$ and the solid angle $\Omega_{M}$.

From Eqs. (1) and (2) we see that the product of area $A_{M}$ and the solid angle $\Omega_{M}$ is constant, namely

$$
\mathrm{A}_{\mathrm{M}} \Omega_{\mathrm{M}}=\lambda^{2}
$$

which is the basic definition of a spatial mode. The exact shape of modes depends on boundary conditions (e.g. waveguides, laser resonators) and can be changed by optical elements. However, for the use in the following discussions, only the property given in Eq. (3) is relevant and needed. This property means, that a mode occupies in the "phase-space" (transverse position and momentum of the corresponding photons) always the same volume.

\subsection{Temporal modes}

The definition of temporal modes can be based on the Wiener-Khinchin theorem, which relates the temporal coherence function $\Gamma(\tau)$ and the power spectral density $S(v)$, or often simply called the spectrum, by

$$
\Gamma(\tau)=\left\langle\mathrm{V}(\mathrm{t}+\tau) \mathrm{V}^{*}(\mathrm{t})\right\rangle=\int_{-\infty}^{\infty} \mathrm{d} \nu S(\mathrm{v}) \mathrm{e}^{\mathrm{i} 2 \pi \nu \tau},
$$

where $\left\langle\mathrm{V}(\mathrm{t}+\tau) \mathrm{V}^{*}(\mathrm{t})\right\rangle$ is the autocorrelation function of the complex amplitude $\mathrm{V}(\mathrm{t}), \tau$ is the time delay and $v$ is the optical frequency. For a rectangular spectrum

$$
S(v)=\frac{\mathrm{P}_{0}}{\Delta v} \operatorname{rect}\left(\frac{v-v_{0}}{\Delta v}\right)
$$

of width $\Delta v$ we get from Eq. (4) the coherence function

$$
\Gamma(\tau)=\mathrm{P}_{0} \frac{\sin (\pi \Delta v \tau)}{\pi \Delta v \tau}
$$

From Eq. (6) we see that the coherence becomes zero for $\Delta v \tau=1$, which gives

$$
\Delta \mathrm{v}_{\mathrm{M}} \Delta \mathrm{t}_{\mathrm{M}}=1
$$

for the basic definition of a temporal mode. This property means that photons within one temporal mode cannot by distinguished by their energy hv.

For the interested reader and for completeness only, it shown in the following how for more general power spectral density functions $S(v)$ the values of $\Delta v_{M}$ and $\Delta \mathrm{t}_{M}$ have to be defined. These definitions are based on the Wiener-Khinchin theorem, already introduced in Eq. (4). In the general case, the coherence time is defined by

$$
\Delta \mathrm{t}_{\mathrm{M}}=\int_{-\infty}^{\infty} \mathrm{d} \tau|\gamma(\tau)|^{2},
$$

where $\gamma(\tau)=\Gamma(\tau) / \Gamma(0)$ is the normalized coherence function. The corresponding definition of the spectral width is then 


$$
\Delta \mathrm{t}_{\mathrm{M}}=\left(\int_{0}^{\infty} \mathrm{d} v s^{2}(v)\right)^{-1},
$$

where $s(v)=S(v) / \Gamma(0)=S(v) / \mathrm{P}_{0}$ is the power spectral density normalized to the total optical power $\mathrm{P}_{0}$.

2.3 Basic properties of modes (spatial and temporal)

- Light within one mode is coherent (for identical polarization).

- Light from different modes is incoherent, does not interfere (orthogonality).

- Photons within one mode cannot be distinguished (uncertainty relation).

\section{PLANCK'S LAW, LASERS, AND THE QUALITY OF LIGHT}

In the following it will be shown how Planck's law of blackbody radiation, the ratio of the probability for spontaneous and stimulated emission, as introduced by Einstein, and the quality of light in terms of coherence can be easily deduced from and related to the properties of modes.

\subsection{Planck's law of blackbody radiation}

From the basic properties of modes given in Eqs. (3) and (7), we get for the density of modes (per volume $\Delta \mathrm{V}$ and frequency interval $\Delta v$ ) in free space

$$
\mu(v)=\frac{N}{\Delta V \Delta v}=2 \frac{4 \pi}{\Omega_{M}} \frac{\Delta z / c}{(\Delta t)_{M}} \frac{1}{\Delta x \Delta y \Delta z \Delta v}=\frac{8 \pi}{c \lambda^{2}}=\frac{8 \pi v^{2}}{c^{3}},
$$

where $\mathrm{N}$ is the number of modes, the factor 2 accounts for the two orthogonal polarizations, $4 \pi$ is the total solid angle of free space, $\Delta \mathrm{z} / \mathrm{c}$ is the observation time of the light propagating in the $\mathrm{z}$-direction within a box of length $\Delta \mathrm{z}$, and $\mathrm{c}$ is the speed of light. The number of photons per mode $\eta_{\mathrm{T}}$ in thermal equilibrium is given by the Bose-Enstein distribution

$$
\eta_{\mathrm{T}}=\frac{1}{\mathrm{e}^{\mathrm{hv} / \mathrm{kT}}-1}
$$

With $h v$ for the energy of the photons at frequency $v$ we get finally for the energy density in thermal equilibrium

$$
\mathrm{u}_{\mathrm{T}}(v)=\mu(v) \eta_{\mathrm{T}} \mathrm{h} v=\frac{8 \pi v^{2}}{\mathrm{c}^{3}} \frac{\mathrm{hv}}{\mathrm{e}^{\mathrm{h} v / \mathrm{kT}}-1},
$$

which is the well known law of blackbody radiation, first introduced by Planck. Compared with quantum mechanics, $\mu(v)$ represents the density of states and $\eta$ the population (number of particles) of the states. The first part of Eq. (12) holds also for non-thermal radiation and confined modes in optical resonators.

\subsection{Laser: spontaneous and stimulated emission (Einstein)}

Following Einstein the transition probabilities $\mathrm{dW}_{\mathrm{S}}$ for spontaneous emission and $\mathrm{dW}_{21}$ for stimulated emission in a two level system are given by

$$
\mathrm{dW}_{\mathrm{S}}=\mathrm{A}_{21} \mathrm{dt} \quad \text { and } \quad \mathrm{dW}_{21}=\mathrm{B}_{21} \mathrm{u}(\mathrm{v}) \mathrm{dt} .
$$

From considerations of thermal equilibrium (Planck's law, Boltzmann), Einstein found that the coefficients $\mathrm{A}_{21}$ and $\mathrm{B}_{21}$ are related by

$$
\frac{\mathrm{A}_{21}}{\mathrm{~B}_{21}}=\frac{8 \pi \mathrm{h} v^{3}}{\mathrm{c}^{3}}=\mathrm{h} v \mu(v)
$$

Using Eqs. (13) we get for the ratio of stimulated emission, responsible for (coherent) amplification and laser oscillation, and spontaneous (incoherent) emission

$$
\frac{\mathrm{dW}_{21}}{\mathrm{dW}_{\mathrm{s}}}=\frac{\mathrm{B}_{21} \mathrm{u}(\mathrm{v})}{\mathrm{A}_{21}}=\eta
$$


which holds also in the case of non-thermal radiation and confined modes, with $\eta$ being the number of photons in the considered mode. From Eq. (15) we see that break-even between coherent (stimulated) emission and incoherent (spontaneous) emission is obtained for a population of one photon in the considered mode. As a consequence, the quality of light in terms of coherence is characterized by the number of photons per mode with $\eta(v)<1$ for (incoherent) thermal light and $\eta(v)>1$ for (coherent) laser light. We note that for optical frequencies $\left(v>3 \cdot 10^{14} \mathrm{~Hz}\right)$ and reasonable temperatures $\left(\mathrm{T}<5 \cdot 10^{3} \mathrm{~K}\right)$ at thermal equilibrium $\eta_{\mathrm{T}} \ll 1$ (Bose-Einstein).

\subsection{Coherence and quality of light}

The quality of light in terms of coherence can be defined by the coherent energy, which is the energy per mode in space and time. From the definition of temporal modes in Eq. (7) we get for the coherent energy in time

$$
\mathrm{E}_{\mathrm{coh}}=\mathrm{P}_{\mathrm{V}} \Delta \mathrm{v}_{\mathrm{M}} \Delta \mathrm{t}_{\mathrm{M}}=\mathrm{P}_{\mathrm{V}},
$$

where $\mathrm{P}_{\mathrm{v}}=\mathrm{dP} / \mathrm{d} v=S(v)$ is the spectral power, or power spectral density, which is assumed to be nearly constant over the considered spectral width $\Delta v$. In conclusion, the temporal quality of light is given by the spectral power. The coherence time $\Delta \mathrm{t}_{\mathrm{M}}$ can by changed (increased) by appropriate optical filtering, changing $\Delta \mathrm{v}_{\mathrm{M}}$, but the coherent energy $\mathrm{E}_{\mathrm{coh}}$ does not change. From the definition of spatial modes in Eq. (3) we get for the coherent power in space

$$
\mathrm{P}_{\text {mode }}=\mathrm{B} \Omega_{\mathrm{M}} \mathrm{A}_{\mathrm{M}}=\mathrm{B} \lambda^{2},
$$

where $B=d P / d A d \Omega$ is the radiance (power per unit area and solid angle), which is assumed to be nearly constant over the considered area A and solid angle $\Omega$. In conclusion, the spatial quality of light is given by the radiance. The intensity $\mathrm{dP} / \mathrm{dA}$ (power per unit area) can be changed by appropriate optical elements (lenses, mirrors), but the coherent power does not change. From Eqs. (16) and (17) we see that the quality of light in space and time is characterized by the number of photons per mode

$$
\eta_{\text {mode }}=\mathrm{E}_{\text {mode }} / \mathrm{hv}=\mathrm{B}_{\mathrm{v}} \lambda^{2} / \mathrm{hv},
$$

where $B_{V}=d B / d v$ is the spectral radiance.

As shown above, the quality of light in terms of coherence, both in space and time, cannot be increased by passive optical elements. Although this fact can be deduced from geometrical optics for its spatial aspect, the concept of modes and number of photons per mode allows a more fundamental understanding through the concept of entropy and its statistical interpretation (entropy as a measure of disorder). Increasing the coherent power or energy means increasing the number of photons per mode, which corresponds to increased order and therefore to decreased entropy, clearly in contradiction to the second law of thermodynamics.

\section{MODES IN FIBERS AND COUPLING EFFICIENCY}

In the following it will be shown how the basic definition of modes, given in Eqs. (3) and (7), can be used to estimate number of accepted modes in a fiber, without being obliged to known the shape of the modes. The same approach is also used to estimate an upper limit for the coupling efficiency of different light sources into fibers and between different types of fibers.

\subsection{Multi-mode and single-mode fibers}

A typical step-index fiber, as shown in Fig. 2, is characterized by the numerical aperture

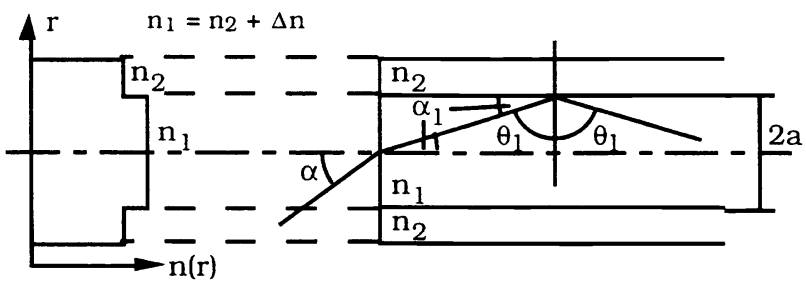

$$
\mathrm{NA}=\sin \alpha_{\mathrm{c}}=\sqrt{\mathrm{n}_{1}^{2}-\mathrm{n}_{2}^{2}}
$$

the corresponding solid angle of acceptance

$$
\Omega_{\mathrm{c}}=\pi(\mathrm{NA})^{2},
$$

Fig. 2. Step-index fiber

and the area of acceptance (cross-section of the core)

$$
\mathrm{A}_{\mathrm{c}}=\pi \mathrm{a}^{2} \text {. }
$$

The number $\mathrm{N}$ of accepted modes can be estimated by comparing the volume in "phase-space" $\Omega_{\mathrm{M}} \mathrm{A}_{\mathrm{M}}$ required by a (spatial) mode, Eq. (3), and the volume offered by the fiber, $\Omega_{c} A_{c}$. The result is 


$$
\mathrm{N}=\frac{\Omega_{\mathrm{c}} \mathrm{A}_{\mathrm{c}}}{\Omega_{\mathrm{M}} \mathrm{A}_{\mathrm{M}}}=\left(\frac{\pi \mathrm{NA} a}{\lambda}\right)^{2}
$$

For single-mode fibers $\mathrm{N} \approx 1$ and for multi-mode fibers $\mathrm{N}>>1$. The estimation from Eq. (22) holds also for graded index fibers or any other type of waveguide. However, to get the accurate number of low order modes the exact shape and cutoff conditions of the modes have to be known.

In the case of multi-mode transmission, the maximum information rate is limited by the spread of the group velocity of the different modes, the modal dispersion. There is spatial multi-mode transmission in multi-mode fibers $(\mathrm{N}>1)$ and temporal multi-mode transmission, if the spectral width $(\Delta \mathrm{v})_{\text {source }}$ of the source is larger than the bandwidth required by the bit length $(\Delta t)_{\text {bit }}$, viz. $(\Delta v)_{\text {source }}(\Delta t)_{\text {bit }}>1$.

\subsection{Energy throughput and coupling efficiency}

The energy throughput of an optical system is limited by the number of accepted modes and the number of photons per mode. Since the number of photons per mode cannot be increased by passive elements, any reduction of the number of modes from one part of an optical system to the next will cause a reduction of the energy throughput. This reduction can be described by a coupling efficiency smaller than one.
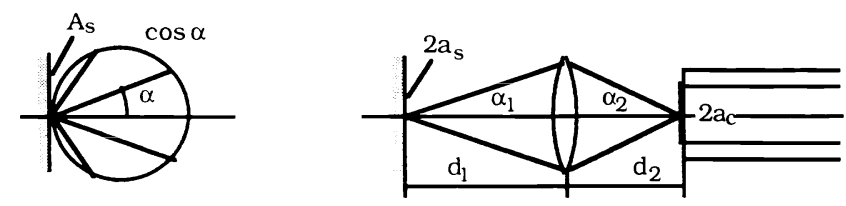

The spatial distribution of the emission of a laser source is characterized by the number of transverse modes and the power per mode. The emission of an incoherent source (LED) is best described by a Lambertian distribution of the radiance (Fig. 3)

Fig. 3. Coupling from a source (LED) to an optical fiber

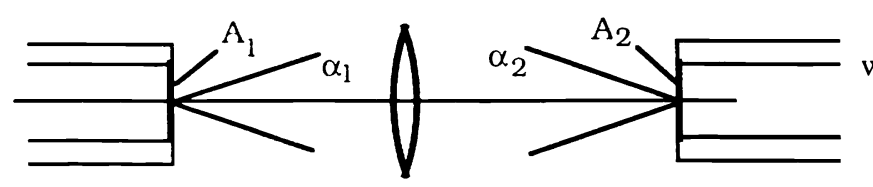

$$
\mathrm{B}(\alpha)=\mathrm{B}_{0} \cos \alpha
$$

with the total power

$$
\mathrm{P}=\mathrm{A}_{\mathrm{s}} \int \mathrm{B}(\alpha) \mathrm{d} \Omega=\pi \mathrm{B}_{0} \mathrm{~A}_{s},
$$

Fig. 4. Coupling between different optical fibers

where $\mathrm{B}_{0}$ is the radiance on axis and $\mathrm{A}_{\mathrm{S}}$ the emitting area.

As shown in Fig. 3. coupling between source and optical fiber can be accomplished by an appropriate relay lens or direct pig-tailing. Using the concept of (spatial) modes, we get for the maximum power coupled into the fiber

$$
\mathrm{P}_{\max }=\mathrm{P}_{\text {mode }} \mathrm{N}_{\text {fibre }}=\mathrm{B}_{0} \pi(\mathrm{NA})^{2} \mathrm{~A}_{\mathrm{c}}, \quad\left(\mathrm{P}_{\max } \leq \mathrm{P}\right),
$$

where $\mathrm{P}_{\text {mode }}$ is the power per mode and $\mathrm{N}_{\text {fibre }}$ is the number of modes accepted by the fiber. For an LED we get from Eq. (17) $P_{\text {mode }}=B_{0} \lambda^{2}(\alpha<<1)$ and $N_{\text {fibre }}$ is given by Eq. (22). The maximum coupled power into a single-mode fiber from an LED is then obviously $\mathrm{P}_{\max }=\mathrm{B}_{0} \lambda^{2}$, with $\mathrm{B}_{0}$ given in Eq. (24). Similarly, we get for the coupling between different fibers (Fig. 4) for the maximum coupled power into the second fiber

$$
\mathrm{P}_{2}=\left(\mathrm{N}_{2} / \mathrm{N}_{1}\right) \mathrm{P}_{1}, \quad\left(\mathrm{P}_{2} \leq \mathrm{P}_{1}\right),
$$

where $P_{1}$ is the output power of the first fiber, and $N_{1}$ and $N_{2}$ are the number of modes in the two fibers, respectively. This consideration holds for any other type of coupling between fibers or parts of an optical system.

\section{COHERENT DETECTION}

Coherent optical detection is employed in all types of interferometry, but also in spectroscopy and communication. It is obvious, that only the mutually coherent parts of the two superposed light waves will contribute to the coherent detection signal. Therefore, the concept of modes is adequate for the description of the performance of coherent detection. In the case of interferometry with diffusely scattering objects (rough surfaces, dispersed particles, etc), the return light from the coherently illuminated object shows speckles (Fig. 5), corresponding to different zones of uncorrelated statistical intensity ad phase. The size of these zones, or speckles, is given by the spatial modes of the scattered light.

From Fig. 5 we can deduce for the coherent power

$$
\mathrm{P}_{\text {coh }}=\frac{1}{2} \mathrm{P}_{\text {mode }}=\frac{\Omega_{0}}{4 \pi} \mathrm{P}_{0}=\frac{\lambda^{2} \mathrm{P}_{0}}{4 \pi \mathrm{A}_{\mathrm{o}}}=\frac{\lambda^{2} \mathrm{I}_{0}}{4 \pi}
$$




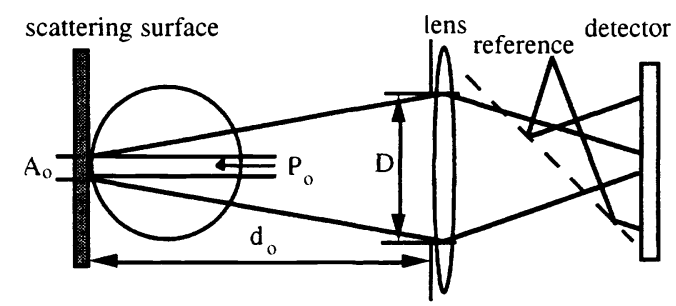

Fig. 5. Speckle interferometry, coherent detection

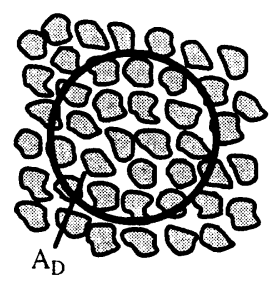

where the factor $1 / 2$ stands for the fact that scattered light will be depolarized, $\Omega_{\mathrm{o}}$ is the solid angle of the spatial modes of the light scattered from the illuminated area $A_{O}, P_{O}$ is the total power and $I_{O}$ the intensity of the coherent illumination. The number of modes (or speckles) collected by the lens depends on its numerical aperture on the object side $\left(\mathrm{NA} \cong \mathrm{D} / 2 \mathrm{~d}_{\mathrm{o}}\right)$.

In the case of shot-noise limited coherent detection, the signal-to-noise ratio (SNR) is equal to the number of detected photons per mode of the scattered light from the object (Poisson statistics), which yields

$$
\mathrm{SNR}=2 \eta_{\mathrm{D}}\left(\mathrm{P}_{\mathrm{coh}} / \mathrm{hv}\right)(\Delta \mathrm{t})_{\mathrm{D}},
$$

where $\eta_{D}$ is the quantum efficiency of the photo-detector and $(\Delta t) D$ the integration time (the factor 2 stands for the so-called coherent detection gain). We see, that the SNR is independent of the number of speckles collected by the detector. If the area $A_{D}$ of the detector is increased, the total detected power will increase, but the modulation depth of the coherent signal (fringe contrast) well decrease accordingly. Increasing the number of detected speckles may help to overcome the electronic noise, but not to increase the shot-noise limited SNR.

\section{CONCLUSIONS}

The examples presented in this paper have shown, that in optics and photonics the concept of modes is well suited to describe emission and absorption, coherence and interference, propagation and dispersion. Besides that, the concept of modes is fundamental in physics (quantum mechanics), and the examples from optics help to understand this concept, because the consequences and properties of modes become visible. These are the reasons why I believe that introducing the concept of modes is most adequate for education in optics and photonics, at least at a university level.

\section{REFERENCES}

Additional information and more details can be found in any textbook on modern optics and photonics, e.g.,

A. Yariv, Optical Electronics in Modern Communications, Oxford University Press, New York, 1997

B.E.A. Saleh and M.C. Teich, Fundamentals of Photonics, John Wiley \& Sons, New York, 1991 